\title{
Nonautonomous Beverton-Holt equations and the Cushing-Henson Conjectures
}

\author{
Saber Elaydi \\ Department of Mathematics \\ Trinity University \\ San Antonio, Texas 78212, USA \\ E-mail: selaydi@trinity.edu \\ http://www.trinity.edu/selaydi
}

\author{
Rober J. Sacker*† \\ Department of Mathematics \\ University of Southern California \\ Los Angeles, CA 90089-2532 USA \\ E-mail: rsacker@math.usc.edu \\ http://math.usc.edu/ rsacker
}

\section{Introduction}

In [3] Jim Cushing and Shandelle Henson published two conjectures (see Section 3) related to the Beverton-Holt difference equation (with growth parameter exceeding one) which said that the B-H equation with periodically varying coefficients (a) will have a globally asymptotically stable periodic solution and (b) the average of the state variable along the periodic orbit will be strictly less than the average of the carrying capacities of the individual maps. They had previously [2] proved both statements for period 2 .

In [4] the authors solved the first conjecture in the affirmative for arbitrary period and for a more general class of maps in a metric state space. In addition they showed that the period of the periodic "geometric cycle", i.e. the projection of the periodic orbit onto the state space, must be a divisor of the period of the underlying system. In [5] the authors solved the second conjecture.

Independently Ryusuke Kon [7], [8] discovered a solution to the second conjecture and in fact proved the result for a wider class of difference equations including the Beverton-Holt equation. Also Kocic [6] has given a solution to the second conjecture.

In this paper we consider the B-H equation with periodic growth parameter as well as periodic carrying capacity. We first give an estimate relating the averages of the state variable and the carrying capacities. This is done by a modification of the proof of Kocic [6]. We then refine the estimate and actually obtain an equality relating the averages, (in the case of period $p=2$ ) thus laying to rest once and for all the $p=2$ case. The general case will be treated elsewhere.

\footnotetext{
*Supported by University of Southern California, Letters Arts and Sciences Faculty Development Grant

${ }^{\dagger}$ Corresponding author
} 
Finally we show in Section 6 that for the Beverton-Holt equation with periodic carrying capacity and constant $\mu>1$, the unique asymptotically stable periodic solution must have minimal period exactly equal to the minimal period of the equation.

\section{Discrete Skew-Product Systems}

Consider the system of difference equations

$$
x_{n+1}=f_{n}\left(x_{n}\right), \quad n \in \mathbb{Z}^{+}
$$

$f_{n}: X \rightarrow X$, where $f_{n}$ is continuous on a metric space $X$. Let $C$ be the space of continuous functions equipped with the topology of uniform convergence on compact subsets of $\mathbb{Z} \times X$. Define the shift map $\sigma: C \rightarrow C$ by $\sigma\left(f_{i}\right)=f_{i+1}$ and let $Y=\operatorname{cl}\left\{f_{n}: n \in \mathbb{Z}\right\}$. Then if $g \neq f_{n}$ for all $n, g \in Y$, we have $f_{n_{i}} \rightarrow g$ for some subsequence $\left\{f_{n_{i}}\right\}$. It is a standard exercise to verify that $\sigma$ is continuous on $C$.

The map $\pi: X \times Y \times \mathbb{Z}^{+} \rightarrow X \times Y$ defines a skew product semi-dynamical system where $\pi(x, g, n)=\left(\Phi(g, n) x, \sigma^{n}(g)\right)$, with $\Phi(g, n)=\sigma^{n-1}(g) \circ \cdots \circ \sigma(g) \circ g$. We define the "fiber", $\mathcal{F}_{g}$ over $g \in Y$ as

$$
\mathcal{F}_{g}=\operatorname{proj}^{-1}(g)
$$

where $\operatorname{proj}$ is the projection: $\operatorname{proj}: X \times Y \rightarrow Y$ given by $\operatorname{proj}(x, y)=y$. The fiber $\mathcal{F}_{g}$ is just a copy of $X$ residing over $g$ in the product space. We will take the liberty to write $\mathcal{F}_{i}$ for $\mathcal{F}_{f_{i}}$ whenever the focus is specifically on the sequence $\left\{f_{i}\right\}$.

Let $c_{r}=\left\{\bar{x}_{0}, \bar{x}_{1}, \ldots, \bar{x}_{r-1}\right\}$ be a cycle of (2.1) in space $X$ with minimal period $r$, where $f_{n+p}=f_{n}$ for all $n \in \mathbb{Z}^{+}$, and $f_{(i+n r) \bmod p}\left(\bar{x}_{i}\right)=\bar{x}_{(i+1) \bmod r}$. Such a cycle "in the space $X$ " was defined to be a "geometric cycle" in [4] to distinguish it from a true cycle (a periodic orbit) in the skew-product semi-dynamical system $\pi$.

Then $c_{r}$ produces an $r$-cycle in the skew-product dynamical system only if $r \geq p$. In fact an $r$-cycle produces an $s=[r, p]$-cycle in the skew-product system as may be seen from the following result.

Lemma 2.1. Let $s=[r, p]$ be the least common multiple of $r$ and $p$. Then the orbit of $\left(c_{0}, f_{0}\right)$ in the skew-product system intersects each fiber $\mathcal{F}_{i}, i=0,1, \ldots, p-1$ in exactly $l=\frac{s}{p}$ points and each of these points is periodic under $\pi$ with period $s$.

Proof. Define $s$ to be the least common multiple of $r$ and $p$, and positive integers $m$ and $\ell$ such that

$$
s \doteq[r, k]=m r=\ell p
$$

Define $\nu_{i}$ to be the number of distinct $c_{i}$ which land in $\mathcal{F}_{i}$ under repeated application of the appropriate maps. First consider $i=0$. Then $\nu_{0}$ is the number of distinct $i, 0 \leq i \leq p-1$, for which

$$
(i+t r) \bmod k=0
$$


or equivalently

$$
i+t r=\rho p \quad \text { for some } \rho=\rho_{t}
$$

where $t$ is the number of executions of the $r$-cycle. Thus $0 \leq t<m-1$ and since $0 \leq i<p \leq r$, it follows that

$$
i+t r<m r=l p
$$

which implies that $0 \leq \rho_{t}<\ell$ in (2.3). Thus, even if it $\rho_{t}$ attains all possible values, we generate at most $\ell$ distinct $i$ values, i.e. $\nu_{0} \leq \ell$. A similar argument shows $\nu_{i} \leq \ell$ for all possible $i$.

From (2.2) we see that each of the fibers $\mathcal{F}_{i}$ contains on average $s / p=l$ points. But if $\nu_{i}<\ell$ on some fiber then $\nu_{j}>\ell$ on some other fiber, a contradiction. Thus $\nu_{i}=l, i=0, \ldots, p-1$.

\section{The Cushing-Henson Conjectures}

Cushing and Henson [3] conjectured that for the $p$-periodic Beverton-Holt equation, $p \geq 2$,

$$
x_{n+1}=\frac{\mu K_{n} x_{n}}{K_{n}+(\mu-1) x_{n}}, \quad \mu>1, K_{n}>0
$$

1. There is a positive $k$-periodic solution $\left\{\bar{x}_{0} \ldots, \bar{x}_{k-1}\right\}$ and it globally attracts all positive solutions

2. The average $\quad a v\left(x_{n}\right) \doteq \frac{1}{k} \sum_{i=0}^{k-1} x_{i}$ satisfies

$$
a v\left(\bar{x}_{n}\right)<a v\left(K_{n}\right)
$$

When the $K_{n}=K$ are all the same, the autonomous case, it is clear that the mapping $x_{n} \rightarrow x_{n+1}$ has a globally (in $\mathbb{R}^{+}$) attracting fixed point $K$ and the "averages" are trivially equal. The interpretation of the second conjecture is that a periodically varying carrying capacity (a fluctuating environment) has a deleterious effect on the average of the state variable along a periodic orbit.

Earlier [2] Cushing and Henson proved both statements for $k=2$.

\section{Extensions}

In this section we assume that both the intrinsic growth rate $\mu$ and the carrying capacity $K$ are periodic of minimal common period $p \geq 2$. The Beverton-Holt equation becomes

$$
x_{n+1}=\frac{\mu_{n} K_{n} x_{n}}{K_{n}+\left(\mu_{n}-1\right) x_{n}}
$$

with $\mu_{n+p}=\mu_{n}$ and $K_{n+p}=K_{n}$, for all $n \in \mathbb{Z}^{+}$.

Equation (4.1) may be written in the form

$$
x_{n+1}=f_{n}\left(x_{n}\right),
$$


where

$$
f_{n}(x)=\frac{\mu_{n} K_{n} x}{K_{n}+\left(\mu_{n}-1\right) x}
$$

Notice that the map

$$
H(x)=f_{p-1} \circ f_{p-2} \circ \cdots \circ f_{0}(x)
$$

defines a discrete semidynamical system on $\mathcal{F}_{0}=\mathbb{R}^{+}$given by

$$
x_{n+1}=H\left(x_{n}\right)
$$

Now

$$
H(x)=\frac{Q_{p-1} L_{p-1} x}{L_{p-1}+E_{p-1} x}
$$

where

$$
\begin{aligned}
L_{p-1} & =K_{p-1} \ldots K_{0} \\
Q_{p-1} & =\mu_{p-1} \ldots \mu_{0} \\
E_{p-1} & =K_{p-1} E_{p-2}+\left(\mu_{p-1}-1\right) \mu_{p-2} \mu_{p-3} \ldots \mu_{0} K_{p-2} K_{p-3} \ldots K_{0}
\end{aligned}
$$

with $E_{0}=\mu_{0}-1$. We thus obtain

$$
E_{p-1}=\left(\prod_{j=0}^{p-2} K_{j+1}\right)\left(\mu_{0}-1\right)+\sum_{s=0}^{p-2}\left[\left(\mu_{s+1}-1\right) \prod_{j=s+1}^{p-2} K_{j+1} \prod_{j=0}^{s} \mu_{j} \prod_{j=0}^{s} K_{j}\right] .
$$

Equation (4.1) has a $p$-periodic solution if and only if $H\left(\bar{x}_{0}\right)=\bar{x}_{0}$ or

$$
\bar{x}_{0}=\frac{Q_{p-1} L_{p-1} \bar{x}}{L_{p-1}+E_{p-1} \bar{x}} .
$$

Hence

$$
\bar{x}_{0}=\frac{L_{p-1}\left(Q_{p-1}-1\right)}{E_{p-1}}
$$

is a point in the unique periodic orbit of equation (4.1). Since $H$ belongs to the class $\mathcal{K}$ defined in $([4])$, this unique periodic orobit is globally asymptotically stable. This establishes the first of the Cushing-Henson conjectures.

Next we turn our attention to the second conjecture. The proof is similar to the one used by Kocic [6]. 
Let $c_{p}=\left\{\bar{x}_{0}, \bar{x}_{1}, \ldots, \bar{x}_{p-1}\right\}$ be the unique periodic orbit of equation (4.1). Then

$$
\begin{aligned}
\operatorname{av}\left(\overline{\mathrm{x}}_{\mathrm{n}}\right) & =\frac{1}{p} \sum_{i=0}^{p-1} \bar{x}_{i}=\frac{1}{p} \sum_{i=0}^{p-1} \bar{x}_{i+1} \\
& =\frac{1}{p} \sum_{i=0}^{p-1} \frac{\mu_{i} K_{i} \bar{x}_{i}}{K_{i}+\left(\mu_{i}-1\right) \bar{x}_{i}} \\
& =\frac{1}{p} \sum_{i=0}^{p-1} \frac{\left(\frac{\mu_{i} K_{i}}{\mu_{i}-1}\right)\left(\frac{\left(\mu_{i}-1\right)}{K_{i}} \bar{x}_{i}\right)}{1+\left(\frac{\left(\mu_{i}-1\right)}{K_{i}} \bar{x}_{i}\right)} \\
& =\frac{1}{p} \frac{\left[\sum_{i=0}^{p-1}\left(\frac{\mu_{i} K_{i}}{\mu_{i}-1}\right) f\left(\frac{\left(\mu_{i}-1\right)}{K_{i}} \bar{x}_{i}\right)\right]}{p-1} \sum_{i=0}^{p-1} \frac{\mu_{i} K_{i}}{\mu_{i}-1}
\end{aligned}
$$

where $f(x)=\frac{x}{1+x}$. Clearly $f$ is concave and thus satisfies Jensen's inequality

$$
f\left(\frac{\sum_{i=0}^{p-1} w_{i} u_{i}}{\sum_{i=0}^{p-1} w_{i}}\right)>\frac{\sum_{i=0}^{p-1} w_{i} f\left(u_{i}\right)}{\sum_{i=0}^{p-1} w_{i}} .
$$

Letting $w_{i}=\frac{\mu_{i} K_{i}}{\mu_{i}-1}, u_{i}=\frac{\mu_{i}-1}{K_{i}} \bar{x}_{i}$ and applying Jensen's inequality yields

$$
\begin{aligned}
\operatorname{av}\left(\overline{\mathrm{x}}_{\mathrm{n}}\right) & <\frac{1}{p} \sum_{i=0}^{p-1} \frac{K_{i} \mu_{i}}{\mu_{i}-1} f\left(\frac{\sum_{i=0}^{p-1} \frac{K_{i} \mu_{i}}{\left(\mu_{i}-1\right)} \frac{\left(\mu_{i}-1\right)}{K_{i}} \bar{x}_{i}}{\sum_{i=0}^{p-1} \frac{K_{i} \mu_{i}}{\left(\mu_{i}-1\right)}}\right) \\
& =\frac{1}{p} \sum_{i=0}^{p-1} \frac{K_{i} \mu_{i}}{\mu_{i}-1} \cdot \frac{\sum_{i=0}^{p-1} \mu_{i} \bar{x}_{i} / \sum_{i=0}^{p-1} \frac{K_{i} \mu_{i}}{\left(\mu_{i}-1\right)}}{\sum_{i=0}^{p-1} \mu_{i} \bar{x}_{i}} \\
= & \frac{1}{p} \sum_{i=0}^{p-1} \frac{K_{i} \mu_{i}}{\mu_{i}-1} \cdot\left[\frac{K_{i} \mu_{i}}{\left(\mu_{i}-1\right)} \cdot \frac{\frac{1}{p} \sum_{i=0}^{p-1} \mu_{i} \bar{x}_{i}}{\frac{1}{p} \sum_{i=0}^{p-1} \frac{K_{i} \mu_{i}}{\left(\mu_{i}-1\right)}+\frac{1}{p} \sum_{i=0}^{p-1} \mu_{i} \bar{x}_{i}}\right] .
\end{aligned}
$$

Let

$$
T_{p}=\frac{1}{p} \sum_{i=0}^{p-1} \frac{K_{i} \mu_{i}}{\left(\mu_{i}-1\right)}
$$


Then

$$
\begin{aligned}
\operatorname{av}\left(\overline{\mathrm{x}}_{\mathrm{n}}\right) & <T_{p} \cdot \frac{\mu^{*} \mathrm{av}\left(\overline{\mathrm{x}}_{\mathrm{n}}\right)}{T_{p}+\mu_{*} \operatorname{av}\left(\mathrm{x}_{\mathrm{n}}\right)}, \quad \mu^{*}=\max \left(\mu_{n}\right) \quad \mu_{*}=\min \left(\mu_{n}\right) \\
T_{P}+\mu_{*} \operatorname{av}\left(\overline{\mathrm{x}}_{\mathrm{n}}\right) & <\mu^{*} T_{p} \\
\operatorname{av}\left(\overline{\mathrm{x}}_{\mathrm{n}}\right) & <T_{p} \frac{\left(\mu^{*}-1\right)}{\mu_{*}}
\end{aligned}
$$

Thus

$$
\operatorname{av}\left(\overline{\mathrm{x}}_{\mathrm{n}}\right)<\frac{\mu^{*}}{\mu_{*}} \cdot \frac{\left(\mu^{*}-1\right)}{\left(\mu_{*}-1\right)} \operatorname{av}\left(\mathrm{K}_{\mathrm{n}}\right) .
$$

While this expression certainly gives an upper bound on the constant relating the two averages, it suffers from the deficiency that if one of the $\mu$ values is close to unity, the constant becomes very large. In the next section we will refine the estimate for period $p=2$.

\section{$5 \quad$ Refinement of the estimate}

In this section we consider (4.1) in which the period $p=2$.

We make use of the following elementary identity:

Lemma 5.1. Define, for $\alpha, \beta, x, y \in(0, \infty), \alpha+\beta=1$,

$$
\frac{x y}{\alpha x+\beta y}-\beta x-\alpha y=\frac{-\alpha \beta(x-y)^{2}}{\alpha x+\beta y}
$$

Proof Letting $g(x, y)$ represent the left side of (5.1), we have

$$
\begin{aligned}
& (\alpha x+\beta y) g(x, y) \\
= & \left\{\left(1-\alpha^{2}-\beta^{2}\right) x y-\alpha \beta\left(x^{2}+y^{2}\right)\right\} \\
= & -\alpha \beta(x-y)^{2} .
\end{aligned}
$$

The next lemma follows from elementary calculus:

Lemma 5.2. For $x, a>1$ define

$$
u(x, a)=\frac{|a-x|}{a x-1}
$$

Then $u(a, a)=0 \leq u(x, a)<1$.

Letting $x_{f}$ and $\mu_{f}$ denote respectively the stable fixed point and growth rate of a $\mathrm{B}-\mathrm{H}$ function 
$f$, we derive a formula for a fixed point, $x_{f \circ g}$ and $x_{g \circ f}$ of the composition of $2 \mathrm{~B}-\mathrm{H}$ functions

$$
\begin{aligned}
f(x) & =\frac{\mu_{f} x_{f} x}{x_{f}+\left(\mu_{f}-1\right) x}, \quad g(x)=\frac{\mu_{g} x_{g} x}{x_{g}+\left(\mu_{g}-1\right) x} \\
f \circ g & =\frac{\mu_{f} \mu_{g} x_{f} x_{g} x}{x_{f} x_{g}+\left[\left(\mu_{g}-1\right) x_{f}+\left(\mu_{f}-1\right) \mu_{g} x_{g}\right] x} \\
& =\frac{\mu_{f} \mu_{g} x_{f \circ g} x}{x_{f \circ g}+\left(\mu_{f} \mu_{g}-1\right) x}, \text { where } \\
x_{f \circ g} & =\frac{x_{f} x_{g}}{\left(p x_{f}+q x_{g}\right)}, \text { with } p=\frac{\mu_{g}-1}{\mu_{f} \mu_{g}-1} \text { and } q=\frac{\mu_{f}-1}{\mu_{f} \mu_{g}-1} \mu_{g} .
\end{aligned}
$$

Similarly

$$
x_{g \circ f}=\frac{x_{g} x_{f}}{\left(r x_{g}+s x_{f}\right)}, \quad \text { where } r=\frac{\mu_{f}-1}{\mu_{f} \mu_{g}-1}, \text { and } s=\frac{\mu_{g}-1}{\mu_{f} \mu_{g}-1} \mu_{f}
$$

Clearly $p+q=1$ and $r+s=1$. It follows from lemma 5.1 that

$$
x_{f \circ g}=\frac{x_{f} x_{g}}{p x_{f}+q x_{g}} \leq p x_{g}+q x_{f}
$$

with strict inequality if $x_{f} \neq x_{g}$.

Note that from (5.3) the composition of two B-H maps is again a B-H map with $\mu_{f \circ g}=\mu_{f} \mu_{g}$ and $x_{f \circ g}$ given by (5.4) and therefore has a globally asymptotically stable fixed point. In fact the B-H maps with $\mu>1$ form a sub semi-group of the semi-group $\mathcal{K}$ defined in [4].

Let

$$
f_{0}(x)=\frac{\mu_{0} K_{0} x}{K_{0}+\left(\mu_{0}-1\right) x} \quad \text { and } \quad f_{1}(x)=\frac{\mu_{1} K_{1} x}{K_{1}+\left(\mu_{1}-1\right) x}
$$

and let $x_{0}$ and $x_{1}$ be the fixed points of $f_{1} \circ f_{0}$ and $f_{0} \circ f_{1}$, respectively. Then we have

$$
\begin{aligned}
& x_{0}=x_{f_{1} \circ f_{0}}=\frac{\mu_{1}-1}{\mu_{0} \mu_{1}-1} \mu_{0} K_{1}+\frac{\mu_{0}-1}{\mu_{0} \mu_{1}-1} K_{0}-\gamma \mu_{0}\left(K_{0}-K_{1}\right)^{2} \\
& x_{1}=x_{f_{0} \circ f_{1}}=\frac{\mu_{1}-1}{\mu_{0} \mu_{1}-1} K_{1}+\frac{\mu_{0}-1}{\mu_{0} \mu_{1}-1} \mu_{1} K_{0}-\gamma \mu_{1}\left(K_{0}-K_{1}\right)^{2}
\end{aligned}
$$

where

$$
0<\gamma \doteq \frac{\left(\mu_{0}-1\right)\left(\mu_{1}-1\right)}{\left(\mu_{0} \mu_{1}-1\right)^{2}}<1
$$

Adding, we obtain

$$
\begin{aligned}
x_{0}+x_{1} & =(1+\sigma) K_{1}+(1-\sigma) K_{0}-\gamma\left(\mu_{0}+\mu_{1}\right)\left(K_{0}-K_{1}\right)^{2} \\
& =(1+\sigma)\left(K_{1}-\bar{K}\right)+(1-\sigma)\left(K_{0}-\bar{K}\right)+2 \bar{K}-\gamma\left(\mu_{0}+\mu_{1}\right)\left(K_{0}-K_{1}\right)^{2}
\end{aligned}
$$

where

$$
\sigma \doteq \frac{\mu_{1}-\mu_{0}}{\mu_{0} \mu_{1}-1} \quad \text { and } \quad \bar{K} \doteq \frac{\left(K_{0}+K_{1}\right)}{2}
$$


Thus,

$$
x_{0}+x_{1}=2 \bar{K}+\sigma\left(K_{0}-K_{1}\right)-\gamma\left(\mu_{0}+\mu_{1}\right)\left(K_{0}-K_{1}\right)^{2} .
$$

and finally

$$
\frac{x_{0}+x_{1}}{2}=\frac{K_{0}+K_{1}}{2}+\sigma \frac{K_{0}-K_{1}}{2}-\gamma \frac{\mu_{0}+\mu_{1}}{2}\left(K_{0}-K_{1}\right)^{2}
$$

where $0<\gamma<1$ and from Lemma 5.2, $0 \leq|\sigma|<1$. This equality is the final chapter in the saga of the Cushing and Henson conjecture in the period $p=2$ case !

\section{Remarks on Elaydi-Sacker Theorem}

Consider the $p$-periodic difference equation

$$
x_{n+1}=f_{n}\left(x_{n}\right)
$$

with $f_{n+p}=f_{n}$ for all $n \in \mathbb{Z}^{+}$.

In [4], the authors gave the following result.

Theorem 6.1. Assume that $X$ is a connected metric space and each $f_{n}: X \rightarrow X$ is a continuous map. If $c_{r}=\left\{\bar{x}_{0}, \bar{x}_{1}, \ldots, \bar{x}_{r-1}\right\}$ is a globally asymptotically stable $r$-cycle in $X$, i.e. a geometric $r$-cycle of equation (6.1), then $r$ divides $p$.

Consider the following periodic Beverton-Holt equation where the growth rate $\mu$ is constant and the $K_{n}$ have minimal period $p$

$$
x_{n+1}=f_{n}(x)=\frac{\mu K_{n} x_{n}}{K_{n}+(\mu-1) x_{n}}, \quad K_{n}>0, \quad \mu>1 .
$$

In [4], [5] it was shown that equation (6.2) has a globally asymptotically stable cycle of period $p$.

We now show that in the case of the periodic Beverton-Holt equation (6.2) with constant $\mu$, any geometric cycle must have the same minimal period $p$ as the equation. This is in direct contrast to case in which $\mu$ also has minimal period $p$. In that case we construct an example having a geometric cycle with minimal period $r<p$. Of course $r \mid p$ by Theorem 6.1 due to the known asymptotic stability properties of (6.2).

We first derive a general result for (6.1).

Let $d=(r, p)$ be the greatest common divisor of $r$ and $p, s=[r, p], l=\frac{s}{p}$, and $p=m d$.

We need the following preliminaries from number theory [1].

Definition 6.2. An integer $n$ is called a solution of $r x=b \bmod p$ if $r n=b \bmod p$. 
Theorem 6.3. Let $d=(r, p)$. The congruence

$$
r x=b \bmod p
$$

has a solution if and only if $d \mid b$. If solutions exist, there are precisely d incongruent solutions modulo p, given by

$$
x=x^{*}+\left(\frac{p}{d}\right) t \bmod p \quad(t=0,1, \ldots, d-1)
$$

where $x^{*}$ is any solution of the congruence

$$
\left(\frac{r}{d}\right)=\left(\frac{b}{d}\right) \bmod \left(\frac{p}{d}\right) .
$$

We are now ready to present the main result in this section.

Theorem 6.4. Let $c_{r}=\left\{\bar{x}_{0}, \bar{x}_{1}, \ldots, \bar{x}_{r-1}\right\}$ be a set of points in a metric space $X$. For (6.1) let $d=(r, p)$ be the greatest common divisor, $s=[r, p], l=\frac{s}{p}$ and $p=m d$. Then the following statements are equivalent.

1. $c_{r}$ is a geometric cycle of minimal period $r$.

2. For $0 \leq i \leq r-1$, and $n=0,1, \ldots, m-1, f_{(i+n d)} \bmod p\left(\bar{x}_{i}\right)=\bar{x}_{(i+1) \bmod r}$.

3. For $0 \leq i \leq r-1$, and $n=0,1, \ldots, m-1$, the graphs of the functions

$f_{i}, f_{(i+d) \bmod p}, f_{(i+2 d)} \bmod p, \ldots, f_{(i+(m-1) d)} \bmod p$ intersect at the $l$ points $\left(\bar{x}_{i}, \bar{x}_{(i+1) \bmod r}\right),\left(\bar{x}_{(i+d) \bmod r},\left(\bar{x}_{(i+1+d) \bmod r}\right), \ldots,\left(\bar{x}_{(i+(l-1) d) \bmod r}, \bar{x}_{(i+(l-1) d+1) \bmod p}\right)\right.$.

Proof. $\quad-1 \Rightarrow 2$ Let $c_{r}=\left\{\bar{x}_{0}, \bar{x}_{1}, \ldots, \bar{x}_{r-1}\right\}$ be a geometric $r$-cycle. Then $f_{(i+n r) \bmod p}\left(\bar{x}_{i}\right)=$ $\bar{x}_{(i+1) \bmod r}$. Let $d=(r, p)$ be the greatest common divisor of $r$ and $p$. Then by Theorem 6.4 the equation $r x=d \bmod p$ has a solution given by (6.4). Similarly $r x=2 d$ $\bmod p, \ldots, r x=(m-1) d \bmod p$ all have solutions given by (6.4). Thus $\{n d \bmod p$ : $\left.n \in \mathbb{Z}^{+}\right\}=\left\{m r \bmod p: m \in \mathbb{Z}^{+}\right\}$. Consequently, for each $n \in \mathbb{Z}^{+}$, there exists $m \in \mathbb{Z}^{+}$ such that $f_{n d \bmod p}=f_{m r} \bmod p$. Hence $f_{n d \bmod p}\left(\bar{x}_{i}\right)=\bar{x}_{(i+1) \bmod r}$. To this end we have verified Statement 2 for $i=0$. By a similar argument one may verify Statement 2 for $i>0$.

- $2 \Rightarrow 3$ Let $i=0$. Then Statement 2 yields for $n=0, f_{0}\left(\bar{x}_{0}\right)=\left(\bar{x}_{1}\right)$; for $n=1, f_{d}\left(\bar{x}_{0}\right)=$ $\left(\bar{x}_{1}\right) ; \ldots$; for $n=m-1, f_{(m-1) d}\left(\bar{x}_{0}\right)=\left(\bar{x}_{1}\right)$. Similar analysis may be done for $i=$ $d, 2 d, \ldots,(\ell-1) d$. In particular let $i=(\ell-1) d$. Then Statement 2 yields for $n=$ $0, f_{(\ell-1) d}\left(\bar{x}_{(\ell-1) d}\right)=\bar{x}_{\ell d m o d r}=\bar{x}_{0}$; for $n=1, f_{\ell d}\left(\bar{x}_{(\ell-1) d}\right)=\bar{x}_{0} ; \ldots$; for $n=m-1$, $f_{(\ell-2) d}\left(\bar{x}_{(\ell-1) d}=\bar{x}_{0}\right.$.

This completes the proof of Statement 3. 
- $3 \Rightarrow 1$ From Statement 3 we conclude that each fiber $\mathcal{F}_{i}, i=0,1, \ldots, p-1$, contains $\ell$ distinct points $\bar{x}_{i}, \bar{x}_{(i+d) \text { modr }}, \ldots, \bar{x}_{(i+(\ell-1) d) m o d r}$. Hence those $p$ fibers $\mathcal{F}_{i}, i=0,1, \ldots, p-1$ contain a total of $\ell p$ ponits, not necessarily distinct, from the set $c_{r}$. However, the fibers $\mathcal{F}_{i}, \mathcal{F}_{(i+d) \text { modp }}, \ldots, \mathcal{F}_{(i+(m-1) d) \text { modp }}$ contain the same subset of those $\ell p$ points. Hence there are exactly $\frac{\ell p}{m}=\ell d=r$ points that constitute the $r$-geometric cycle $c_{r}=\left\{\bar{x}_{0}, \bar{x}_{1}, \ldots, \bar{x}_{r-1}\right\}$. This completes the proof of the theorem.

\section{$\underline{\text { Remarks }}$}

1. If $r=4, p=6$ then $d=(r, p)=2, m=3, l=2$. If $c_{r}=\left\{\bar{x}_{0}, \bar{x}_{1}, \bar{x}_{2}, \bar{x}_{3}\right\}$ is a given geometric $r$-cycle, then we can infer from Theorem 6.4

(a) $f_{0}\left(\bar{x}_{0}\right)=\bar{x}_{1}, f_{2}\left(\bar{x}_{0}\right)=\bar{x}_{1}, f_{4}\left(\bar{x}_{0}\right)=\bar{x}_{1}$

(b) $f_{1}\left(\bar{x}_{1}\right)=\bar{x}_{2}, f_{3}\left(\bar{x}_{1}\right)=\bar{x}_{2}, f_{5}\left(\bar{x}_{1}\right)=\bar{x}_{2}$

(c) $f_{2}\left(\bar{x}_{2}\right)=\bar{x}_{3}, f_{4}\left(\bar{x}_{2}\right)=\bar{x}_{3}, f_{6}\left(\bar{x}_{2}\right)=f_{0}\left(\bar{x}_{2}\right)=\bar{x}_{3}$

(d) $f_{3}\left(\bar{x}_{3}\right)=\bar{x}_{4}=\bar{x}_{0}, f_{5}\left(\bar{x}_{3}\right)=\bar{x}_{0}, f_{7}\left(\bar{x}_{3}\right)=f_{1}\left(\bar{x}_{3}\right)=\bar{x}_{0}$

From the above four observations, we conclude that the graphs of the maps $f_{0}, f_{2}, f_{4}$ intersect at the points $\left(\bar{x}_{0}, \bar{x}_{1}\right),\left(\bar{x}_{2}, \bar{x}_{3}\right)$, while the map $f_{1}, f_{3}, f_{5}$ intersect at $\left(\bar{x}_{1}, \bar{x}_{2}\right)$, $\left(\bar{x}_{3}, \bar{x}_{0}\right)$

2. If $r \mid p$, then $d=r$, and $m=\frac{p}{r}$. For example, if $r=3$, and $p=6$, then $d=3, m=2$, $l=1$. Hence the graphs of the maps $f_{0}, f_{3}$ intersect at the points $\left(\bar{x}_{0}, \bar{x}_{1}\right) ; f_{1}, f_{4}$ intersect at $\left(\bar{x}_{1}, \bar{x}_{2}\right) ; f_{2}, f_{5}$ intersect at $\left(\bar{x}_{2}, \bar{x}_{0}\right)$.

Applying Theorem 6.4 to the first periodic Beverton-Holt equation (6.2) yields the following result.

Corollary 6.5. If equation (6.2) is of minimal period p, then there are no nontrivial geometric cycles of period less than $p$. Furthermore, if $\mu>1$, then there is a unique globally asymptotically stable geometric cycle of minimal period $p$.

Proof. Suppose that equation (6.2) has a geometric cycle $c_{r}=\left\{\bar{x}_{0}, \bar{x}_{1}, \ldots, \bar{x}_{r-1}\right\}$ of period $r$, and let $d=(r, p), p=m d$. Then by Theorem 6.4, the graphs of the maps $f_{0}, f_{d}, \ldots, f_{(m-1) d}$ must intersect at the points $\left(\bar{x}_{0}, \bar{x}_{1}\right),\left(\bar{x}_{d}, \bar{x}_{d+1}\right), \ldots,\left(\bar{x}_{(l-1) d}, \bar{x}_{(l-1) d+1}\right)$. Notice that if $f_{i} \neq f_{j}$, $i \neq j$, then the maps $f_{i}$ and $f_{j}$ intersect only at the common fixed point 0 which is not part of any geometric cycle. This implies that $f_{0}=f_{d}=f_{2 d}=\cdots=f_{m d}=f_{0}$.

Similarly, one may show that $f_{1}=f_{d+1}=f_{2 d+1}=\cdots=f_{m d+1}=f_{1}$, etc.

This shows that equation (6.2) is of minimal period $d$, a contradiction. The second statement now follows from [4]. 
To this end we have shown that when the intrinsic growth rate $\mu>1$ is fixed and the carrying capacity $K_{n}$ is periodic, we have no geometric cycles of periods less than the minimal period of the $K_{n}$.

Let us now assume that both $\mu_{n}$ and $K_{n}$ to be of common minimal period $p$, as in equation (4.1). Then we may have geometric cycles of periods less than $p$ as may be illustrated by the following example.

Example 6.6. Consider equation (4.1) with $\mu_{0}=3, K_{0}=1 ; \mu_{1}=4, K_{1}=\frac{6}{17} ; \mu_{2}=2, K_{2}=2$; $\mu_{3}=5, K_{3}=\frac{4}{11}$. Then $c_{2}=\left\{\frac{2}{5}, \frac{2}{3}\right\}$ is a geometric cycle of period 2 .

In contrast to the situation when $\mu$ is fixed, the graphs of the maps $f_{0}$ and $f_{2}$ intersect at the point $\left(\frac{2}{5}, \frac{2}{3}\right)$, while the graphs of the maps $f_{1}$ and $f_{3}$ intersect at the point $\left(\frac{2}{3}, \frac{2}{5}\right)$.

The following technical lemma gives necessary and sufficient conditions under which a geometric cycle of period less than the period of the system is present.

Lemma 6.7. If $r \mid p$, then the following statements are equivalent.

(a) Equation (4.1) has a periodic orbit with a minimal period $r$,

(b) $\frac{L_{p-1}\left(Q_{p-1}-1\right)}{E_{p-1}}=\frac{L_{r-1}\left(Q_{r-1}-1\right)}{E_{r-1}}$,

(c) $\frac{L_{p-1}\left(Q_{p-1}-1\right)}{E_{p-1}}=\frac{K_{0} K_{r}\left(\mu_{r} \mu_{0}\right)}{\mu_{0} K_{0}\left(\mu_{r}-1\right)-\mu_{r} K_{r}\left(\mu_{0}-1\right)}$.

\section{References}

[1] A. Adler and J. Coury, The theory of numbers, Jones and Bartlett Publishers, Boston, 1995.

[2] J.M. Cushing and S.M. Henson. Global dynamics of some periodically forced, monotone difference equations. J. Difference Eq. and Appl., 7:859-872, 2001.

[3] J.M. Cushing and S.M. Henson. A periodically forced Beverton-Holt equation. J. Difference Eq. and Appl., 8:1119-1120, 2002.

[4] S. Elaydi and R.J. Sacker, Global stability of periodic orbits of nonautonomous difference equations and population biology, J. Differential Equations (to appear) doi:10.1016/j.jde.2003.10.024

[5] S. Elaydi and R.J. Sacker, Global stability of periodic orbits of nonautonomous difference equations in population biology and the Cushing-Henson conjectures, Proceedings of the 8th International Conference on Difference Equations, Brno, 2003.

[6] V.L., Kocic, A note on the Cushing-Henson conjectures (this issue).

[7] R. Kon, A note on attenuant cycles of population models with periodic carrying capacity J. Difference Eq. Appl. 10(8) (2004), 791-793. 
[8] R. Kon, Attenuant cycles of population models with periodic carrying capacity J. Difference Eq. Appl. (to appear). 